\title{
PL-SUBMANIFOLDS AND HOMOLOGY CLASSES OF A PL-MANIFOLD*)
}

\author{
MASAHISA ADACHI \\ Dedicated to Professor K. Noshino for his 60 th birthday
}

This paper is devoted to the problem of the realisation of homology classes of a $P L$-manifold by $P L$-submanifolds.

The present study is founded on the consideration of Thom complexes $M\left(P L_{k}\right), M\left(S P L_{k}\right)$ for $P L$-microbundles which is defined by $\mathrm{R}$. Williamson [5]. We shall apply Thom's method [4] to PL-manifolds.

The author is grateful to Professors R. Shizuma and K. Shiraiwa for their kind criticisms.

\section{Generalities}

Following Milnor [3] and Williamson [5] we shall work in the category of locally finite simplicial complexes and piecewise linear maps (briefly, $P L$ maps).

A mapping $F: K \rightarrow L$ between locally finite simplicial complexes is $P L-m a p$, if there exists a rectilinear subdivision $K^{\prime}$ of $K$ so that $f$ maps each simplex of $K^{\prime}$ linearly into a simplex of $L$.

Let $X$ be a locally finite simplicial complex and $Y$ be a closed subspace of it. Then we shall say that $Y$ is a $P L$-subspace of $X$, if $Y$ can be triangulated so that the inclusion $i: Y \rightarrow X$ is a $P L$-map. It follows that some subdivision of $Y$ is a subcomplex of some subdivision of $X$ (cf. Williamson [5], §1). Given two such triangulations the identity is a $P L$-homeomorphism from one to the other.

Let $V^{n}$ be a closed $P L$-manifold ${ }^{1 \prime}$ of dimension $n$. Then we shall say that $W^{p}$ is a $P L$-submanifold of dimension $p$, if $W^{p}$ is a closed $P L$-manifold of

Received March 15, 1966.

*) This work is partially supported by Yukawa Fellowship.

1) By a $P L$-manifold we shall mean a combinatorial manifold. 
dimension $p$ and a $P L$-subspace of $V^{n}$.

In the following we suppose that $V^{n}$ is a closed $P L$-manifold of dimension $n$. Let $W^{p}$ be a $P L$-submanifold of dimension $p$. The inclusion map $i: W^{p} \rightarrow V^{n}$ induces the homomorphism $i_{*}: H_{p}\left(W^{p}, Z_{2}\right) \rightarrow H_{p}\left(V^{n}, Z_{2}\right)$. Let $z \subseteq H_{p}\left(V^{n}, Z_{2}\right)$ be the image by $i_{*}$ of the fundamental class $w$ of the $P L$-manifold $W^{p}$. Then we say that the homology class $z$ is realized by the $P L$-submanifold $W^{p}$. Let $V^{n}$ be oriented, and $W^{p}$ be an oriented $P L$-submanifold of dimension $p$. The inclusion map $i: W^{p} \rightarrow V^{n}$ induces the homomorphism $i_{*}: H_{p}\left(W^{p}, Z\right) \rightarrow H_{p}\left(V^{n}, Z\right)$. Let $z \in H_{p}\left(V^{n}, Z\right)$ be the image by $i_{*}$ of the fundamental class $w$ of the oriented $P L$-manifold $W^{p}$. Then we say that the homology class $z$ is realized by the oriented $P L$-submanifold $W^{p}$.

Here the following questions are considered : Let a homology class $z \bmod$ 2 of the $P L$-manifold $V^{n}$ be given. Is it realisable by a $P L$-submanifold?; Let an integral homology class $z$ of the oriented $P L$-manifold $V^{n}$ be given. Is it realisable by an oriented $P L$-submanifold?

\section{Thom complexes $M\left(P L_{k}\right), M\left(S P L_{k}\right)$}

We shall recall the definition of Thom complexes for $P L$-microbundles (cf. Williamson [5], §4). Let $\xi$ be a $P L$-microbundle:

$$
\xi: B(\xi) \stackrel{i \xi}{\longrightarrow} E(\xi) \stackrel{j \xi}{\longrightarrow} B(\xi) .
$$

Let $E$ be an open neighborhood of $i \xi(B(\xi))$ in $E(\xi)$ such that $E(\xi)-E$ is a $P L$-subspace of $E(\xi)$. If $E(\xi)-E$ is a strong deformation retract of $E(\xi)-i \xi(B(\xi))$, we shall say that $E$ is an admissible neighborhood in Williamson's sense. Then we call the quotient space formed by collapsing $E(\xi)-E$ to a point $*$ a Thom complex of $\xi$ (although it may not be locally finite at $*$ ) and denote it by $T(\xi)$ or $T_{F}(\xi)$. We point out that $T_{E}(\xi)-i \xi(B(\xi))$ is contractible.

Let $U$ be any neighborhood of $i_{\xi}(B(\xi))$ in $E(\xi)$. Then there exists an admissible neighborhood $E$ in Williamson's sense such that $E$ is open and $\bar{E} \subset U$. Moreover, the homotopy type of $T_{E}(\xi)$ does not depend on the particular choice of an admissible neighborhood $E$ (cf. Williamson [5], §4).

We know that for each $n$ there exists a universal $P L$-microbundle for fibre dimension $n$

$$
\gamma\left(P L_{n}\right): B\left(P L_{n}\right) \stackrel{i_{n}}{\longrightarrow} E\left(P L_{n}\right) \stackrel{j_{n}}{\longrightarrow} B\left(P L_{n}\right)
$$


and a universal orientable $P L$-microbundle for fibre dimension $n$

$$
r\left(S P L_{n}\right): B\left(S P L_{n}\right) \stackrel{i_{n}}{\longrightarrow} E\left(S P L_{n}\right) \stackrel{j_{n}}{\longrightarrow} B\left(S P L_{n}\right)
$$

(cf. Milnor [3], §5, Williamson [5], §2). For $T\left(r\left(P L_{n}\right)\right), T\left(r\left(S P L_{n}\right)\right)$ we write $M\left(P L_{k}\right), M\left(S P L_{k}\right)$ respectively.

Let $\xi$ be a $P L$-microbundle of dimension $n$. A $P L$-microbundle $\xi$ is considered as a topological microbundle. Therefore, by Kister [1], there exists an admissible neighborhood $E_{1}(\xi)$ of $i_{\xi}(B(\xi))$ in Kister's sense such that $\left\{E_{1}(\xi), j_{\xi} \mid E_{1}(\xi), B(\xi)\right\}$ is a fibre bundle with fibre $R^{n}$ and structure group $H_{0}(n)$. We have the Thom isomorphism

$$
\varphi_{\xi}^{*}: H^{0}\left(B(\xi), Z_{2}\right) \longrightarrow H^{n}\left(E_{1}(\xi), E_{1}(\xi)-i_{\xi}(B(\xi)) ; Z_{2}\right),
$$

(cf. Milnor [2]). As is remarked above, there exists an admissible neighborhood $E$ of $i \xi(B(\xi))$ in Williamson's sense such that $E$ is open and $\bar{E} \subset E_{1}(\xi)$. Now we consider $n$-th cohomology group of Thom complex $T_{E}(\xi)$ :

$$
\begin{aligned}
H^{n}\left(T_{E}(\xi), Z_{2}\right) & =H^{n}\left(E(\xi) / E(\xi)-E ; Z_{2}\right) \\
& \cong H^{n}\left(E(\xi), E(\xi)-E ; Z_{2}\right) \\
& \cong H^{n}\left(E(\xi), E(\xi)-i_{\xi}(B(\xi)) ; Z_{2}\right) \\
& \cong H^{n}\left(E_{1}(\xi), E_{1}(\xi)-i_{\xi}(B(\xi)) ; Z_{2}\right),
\end{aligned}
$$

where the last isomorphism is the excision. We shall denote this isomorphism by ${ }_{C E}$. Composing two isomorphisms $\varphi_{:}^{*}$ and $C_{E}$, we have

$$
\epsilon_{E}^{\circ} \varphi_{:}^{*}: H^{\circ}\left(B(\xi), Z_{2}\right) \longrightarrow H^{n}\left(T_{E}(\xi), Z_{2}\right) .
$$

Let $\omega$ denote the unit of the cohomology ring $H^{*}\left(B(\xi), Z_{2}\right)$. The cohomology class $U_{\xi} \in H^{n}\left(T_{E}(\xi), Z_{2}\right)$ defined by

$$
U_{\xi}=\imath F^{\circ} \varphi^{*}(\omega)
$$

will be called the fundamental class of Thom complex $T_{E}(\xi)$. In the case where $\xi$ is orientable, we have the Thom isomorphism

$$
\varphi_{\xi}^{*}: H^{0}(B(\xi), Z) \rightarrow H^{n}\left(E_{1}(\xi), E_{1}(\xi)-i \xi(B(\xi)) ; Z\right)
$$

and the fundamental class $U_{\xi} \in H^{n}\left(T_{E}(\xi), Z\right)$, in quite an analogous way (cf. Milnor [2]).

We shall denote by $U_{n}$ the fundamental classes of Thom complexes $M\left(P L_{n}\right)$ and $M\left(S P L_{n}\right)$, and $\varphi_{n}^{*}$ the Thom isomorphisms of universal $P L$-microbundles $r\left(P L_{n}\right)$ and $Y\left(S P L_{n}\right)$. 


\section{Fundamental theorem}

Definition. We say that a cohomology class $u \in H^{k}\left(A, Z_{2}\right)$ of a space $A$ is $P L_{k}$-realisable, if there exists a mapping $f: A \rightarrow M\left(P L_{k}\right)$ such that $u$ is the image, for the homomorphism $f^{*}$ induced by $f$, of the fundamental class $U_{k}$ of the Thom complex $M\left(P L_{k}\right)$. We say that a cohomology class $u \in H^{k}(A, Z)$ of a space $A$ is $S P L_{k}$-realisable, if there exists a mapping $f: A \rightarrow M\left(S P L_{k}\right)$ such that $u$ is the image, for the homomorphism $f^{*}$ induced by $f$, of the fundamental class $U_{k}$ of the Thom complex $M\left(S P L_{k}\right)$.

Then we have the following

THEOREM. Let $V^{n}$ be a closed PL-manifold of dimension $n$.

a) In order that a homology class $z \in H_{n-k}\left(V^{n}, Z_{2}\right) k>0$, can be realized by a PL-submanifold $W^{n-k}$ which has a normal PL-microbundle in $V^{n}$, it is necessary and sufficient that the cohomology class $u \in H^{k}\left(V^{n}, Z_{2}\right)$, corresponding to $z$ by the Poincaré duality, is $P L_{k}$-realisable.

b) Let $V^{n}$ be oriented. In order that a homology class $z \in H_{n-k}\left(V^{n}, Z\right), k>0$, can be realized by an oriented $P L$-submanifold $W^{n-k}$ which has an orientable normal $P L$-microbundle in $V^{n}$, it is necessary and sufficient that the cohonology class $u \in H^{k}\left(V^{n}, Z\right)$, corresponding to $z$ by the Poincaré duality, is $S P L_{k}$-realisable.

Proof. We shall prove the case a) of the theorem. The case b) can be proved quite in parallel with the case a).

i) Necessity. Suppose that there exists a $P L$-submanifold $W^{n-k}$ in $V^{n}$ which have a normal $P L$-microbundle of dimension $k$

$$
\nu: B(\nu) \stackrel{i_{\nu}}{\longrightarrow} E(\nu) \stackrel{j_{\nu}}{\longrightarrow} B(\nu)=W^{n-k} .
$$

The normal $P L$-microbundle $\nu$ is induced from the universal $P L$-microbundle

$$
r\left(P L_{k}\right): B\left(P L_{k}\right) \stackrel{i_{k}}{\longrightarrow} E\left(P L_{k}\right) \stackrel{j_{k}}{\longrightarrow} B\left(P L_{k}\right)
$$

by a mapping $f: W^{n-k} \rightarrow B\left(P L_{k}\right)$. Therefore, there exists a mapping $\bar{f}: E(\nu)$ $\rightarrow E\left(P L_{k}\right)$ such that the following diagram

$$
\begin{aligned}
E(\nu) \stackrel{\bar{f}}{\longrightarrow} & E\left(P L_{k}\right) \\
j \downarrow \downarrow & \quad \downarrow_{k} \\
W^{n-k}= & B(\nu) \stackrel{f}{\rightarrow} B\left(P L_{k}\right)
\end{aligned}
$$


is commutative. The universal $P L$-microbundle $Y\left(P L_{k}\right)$ admits an admissible fibre bundle

$$
r_{1}\left(P L_{k}\right)=\left\{E_{1}\left(P L_{k}\right), j_{k} \mid E_{1}\left(P L_{k}\right), B\left(P L_{k}\right), R^{k}, H_{0}(k)\right\}
$$

in Kister's sense (cf. Kister [1]). Moreover, by the uniqueness of the admissible fibre bundle (cf. Kister [1]), the induced bundle $f^{*} \Upsilon_{1}\left(P L_{k}\right)$ is an admissible fibre bundle

$$
\left\{E_{1}(\nu), j_{\nu} \mid E_{1}(\nu), B(\nu), R^{k}, H_{0}(k)\right\}
$$

of the normal $P L$-microbundle $\nu$. Since $\bar{f}$ maps $E(\nu)-i_{\nu}(B(\nu))$ into $E\left(P L_{k}\right)-$ $i_{k}\left(B\left(P L_{k}\right)\right)$, the following diagram

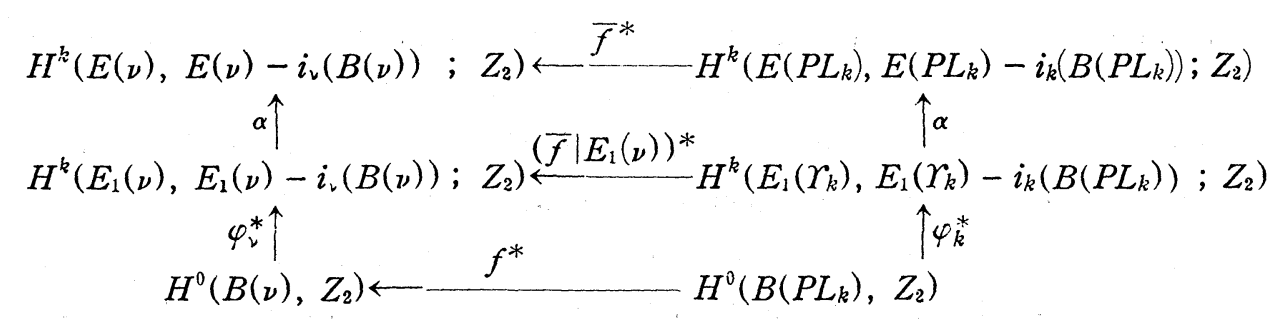

is commutative, where $\alpha$ are the excision isomorphisms (cf. Milnor [2]), and $E_{1}\left(Y_{k}\right)$ denotes $E_{1}\left(P L_{k}\right)$.

Let $E_{k}$ be an admissible neighborhood of $i_{k}\left(B\left(P L_{k}\right)\right)$ in $E\left(P L_{k}\right)$. Let us denote by $g: E(\nu) \rightarrow M\left(P L_{k}\right)$ the composite map, $p \circ \bar{f}$ of $\bar{f}: E(\nu) \rightarrow E\left(P L_{k}\right)$ and the natural projection $p: E\left(P L_{k}\right) \rightarrow E\left(P L_{k}\right) / E\left(P L_{k}\right)-E_{k}=M\left(P L_{k}\right)$. Now we can define mapping $\bar{g}: V^{n} \rightarrow M\left(P L_{k}\right)$ such that $\bar{g} \mid E(\nu)=g$; it is sufficient to map $V^{n}-E(\nu)$ to the point $*$. Then we have the following commutative diagram:

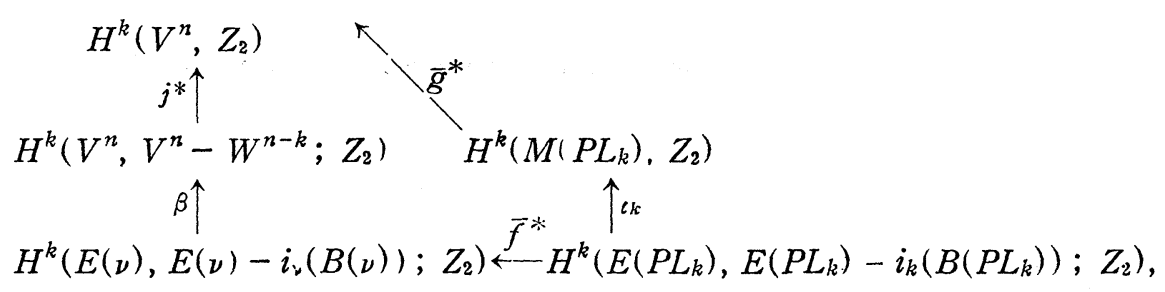

where $j^{*}$ is the relativisation and $\beta$ is the excision isomorphism.

Then we have

$$
\begin{aligned}
\bar{g}^{*}\left(U_{k}\right) & =\bar{g}^{*} \circ \iota_{k} \circ \alpha \circ \varphi_{k}^{*}(\omega) \\
& =j^{*} \circ \beta \circ \alpha \circ \varphi_{\nu}^{*}(\omega) \\
& =\psi\left(i_{W}\right)(\omega),
\end{aligned}
$$


where $\psi\left(i_{W}\right)$ is the Gysin homomorphism of the inclusion map $i_{W}: W^{n-k} \rightarrow V^{n}$. Therefore,

$$
\begin{aligned}
\bar{g}^{*}\left(U_{k}\right) & =D_{r} \circ\left(i_{W}\right)_{*} \circ D_{W}(\omega) \\
& =D_{r} \circ\left(i_{W}\right)_{*}(w) \\
& =D_{r}(z)=u,
\end{aligned}
$$

where $D_{V}$ and $D_{w}$ are the Poincaré dualities of $V^{n}$ and $W^{n-k}$, respectively.

ii) Sufficiency. Suppose that there exists a mapping $f$ of $V^{n}$ into $M\left(P L_{k}\right)$ such that $f^{*}\left(U_{k}\right)=u$. The Thom complex $M\left(P L_{k}\right)$, deprived the point $*$, is considered as a locally finite simplicial complex, and the $P L$-subspace $B\left(P L_{k}\right)$ has the normal $P L$-microbundle $r\left(P L_{k}\right)$ in $M\left(P L_{k}\right)-*$. By the theorem 3.3.1. in Williamson [5], we have a mapping $f_{1}$, homotopic to $f, t$-regular for $(\nu$, $\left.r\left(P L_{k}\right)\right)$, where $\nu$ is a normal $P L$-microbundle of $f_{1}^{-1}\left(B\left(P L_{k}\right)\right)$ in $V^{n}$. However, by the lemma 4.2. in Williamson [5], $f_{1}^{-1}\left(B\left(P L_{k}\right)\right)$ is a $P L$-submanifold $W^{n-k}$ in $V^{n}$. Moreover, by the definition of $t$-regularity, the induced $P L$-microbundle $f_{1}^{*} r\left(P L_{k}\right)$ is isomorphic to $\nu$. We know $f_{1}^{*}\left(U_{k}\right)=f^{*}\left(U_{k}\right)=u$. Then, as in the case i), we can see that the $P L$-submanifold $W^{n-k}$ realizes the homology class $z$, corresponding to $u$ by the Poincaré duality.

\section{REFERENCES}

[1] J. Kister, Microiswdles are fibre bundles, Ann. of Math., 80 (1964), 190-199.

[2] J. Milnor, Lectures Laracteristic classes, Mimeographed Note, Princeton University, 1957-58.

[3] J. Milnor, Microbundles and differentiable structures, Mimeographed Note, Princeton University, 1961.

[4] R. Thom, Quelques propriétés globales des variétés différentiables, Comment. Math. Helv., 28 (1954), 17-86.

[5] R. Williamson, Cobordism of combinatorial manifolds, Ann. of Math., 83 (1966), 1-33.

Mathematical Institute

Nagoya University 